\title{
ANNEAUX DE VALUATION DISCRETE COMPLETS NON COMMUTATIFS
}

\author{
BY \\ ROBERT VIDAL ${ }^{1}$
}

\begin{abstract}
On construit, grace au concept de bimodule au sens de M. Artin, une bonne généralisation non commutative de la notion d'anneau de valuation discrète complet; puis on étudie la validité du théorème de structure de I. S. Cohen en égale caractéristique. Lorsque l'anneau de valuation possède un corps de représentants qui est un corps local (commutatif) à corps résiduel de caractéristique nulle et algébriquement clos, on donne une classification des déviations de commutativité engendrées par une dérivation continue de ce corps local. Enfin, on propose une méthode générale de construction d'anneaux sans corps de Cohen en égale caractéristique et l'article se termine par des problèmes ouverts dans cette théorie.
\end{abstract}

Introduction. Dans ce travail, on se propose de définir et d'étudier une classe d'anneaux de valuation discrète complets, essentiellement non commutatifs, qui constitue une bonne généralisation de la situation commutative et prolonge certaines constructions sur les anneaux d'opérateurs différentiels et les anneaux de séries formelles tordues à coefficients dans un corps gauche. Voir, par exemple, [P.M.C.1 et 2].

Dans le $\$ 1$, on donne les définitions et les premières propriétés de ces anneaux; puis on caractérise dans le $\$ 2$ les anneaux qui vérifient le théorème de structure de I. S. Cohen en égale caractéristique. On conjecture alors que, contrairement à ce qui se passe en algèbre commutative, il existe des anneaux de valuation discrète complets, en égale caractéristique, qui ne sont pas des anneaux de Cohen.

Le $\$ 3$ est consacré à l'étude des anneaux de Cohen lorsque le corps de représentants $K$ est un corps local, commutatif, à corps résiduel $k$ de caractéristique nulle et algébriquement clos et que la déviation de commutativité $s$ est engendrée par une dérivation continue $\delta$ de $K$; c'est à dire: $s=\Sigma_{n \geqslant 0} \delta^{n} X^{n}$. Un tel anneau est noté: $K[[X, \delta]]$ et appelé anneau de Cohen à dérivation. Le théorème central donne une classification de ces anneaux en fonction de la valuation et du résidu d'une forme différentielle canoniquement associée à la dérivation considérée.

Dans le $\S 4$, on applique certains résultats du $\$ 3$ à l'étude des anneaux: $\bar{K}[[X, \delta]]$, où $\bar{K}$ désigne la clôture algébrique (corps de Puiseux) du corps local $K$. On obtient ainsi une première généralisation lorsque le corps de représentants est de valuation dense.

Received by the editors May 30, 1980 and, in revised form, September 11, 1980.

1980 Mathematics Subject Classification. Primary 16A04, 16A05, 16A39; Secondary 12F05, 12 F20.

${ }^{1}$ Cet article contient une partie de la Thèse de Doctorat ès Sciences mathématiques soutenue le 16 mars 1978 auprès de l'Université Pierre et Marie Curie-Paris VI-devant le jury composé de: L. Schwartz, M. Artin, M. P. Malliavin, J. P. Lafon, F. Aribaud, M. Guillaume. 
Le $\$ 5$ donne l'idée d'une méthode générale de construction, en égale caractéristique, d'anneaux sans corps de Cohen. Deux exemples concrets sont traités.

L'appendice regroupe un certain nombre de remarques et de problèmes ouverts dans cette théorie ainsi que les directions dans lesquelles ce travail peut être poursuivi.

1. Anneaux de valuation discrète complets. On appelle anneau de valuation discrète complet, un anneau local $\Lambda$, non nécessairement commutatif, d'idéal maximal $\Re$, qui vérifie:

$\Lambda$ est séparé, complet et non discret pour la topologie de Krull.

$\mathfrak{K} / \mathfrak{M}^{2}$ est un $\Lambda$-bimodule simple. (Ce qui signifie que $\mathfrak{K} / \mathfrak{K}^{2}$ est engendré par tout élément non nul de son centralisateur; voir M. Artin [M.A.].)

Soit $\pi$ un relèvement dans $\mathfrak{T}$ d'un élément non nul du centralisateur de $\Re / \Re^{2}$; un tel élément est appelé une uniformisante de $\Lambda$. On a alors

$$
\forall \lambda \in \Lambda, \quad \pi \lambda-\lambda \pi \in \Re^{2} \text { et } \Lambda \pi+\Re^{2}=\pi \Lambda+\Re^{2}=\mathfrak{\pi} \text {. }
$$

L'anneau $\Lambda$ étant séparé et complet, il s'ensuit que: $\Lambda \pi=\pi \Lambda=\mathfrak{N}$, et par itération

$$
\forall n>0, \forall \lambda \in \Lambda, \quad \pi^{n} \lambda-\lambda \pi^{n} \in \mathscr{M}^{n+1} \text { et } \Lambda \pi^{n}=\pi^{n} \Lambda=\mathfrak{K}^{n} .
$$

Soit $o: \Lambda-\{0\} \rightarrow \mathbf{N}$ la fonction d'ordre de l'anneau $\Lambda$ relative à la filtration Th-adique et convenons de poser: $o(0)=+\infty$. On montre, comme dans le cas commutatif que les $\left(\Re^{n}\right)_{n>0}$ sont les seuls idéaux (à droite ou à gauche) non triviaux de l'anneau $\Lambda$ et que tout élément $\lambda$ non nul de $\Lambda$ s'écrit $\lambda=u \pi^{o(\lambda)}=$ $\pi^{o(\lambda)} v$ avec $u, v$ inversibles dans $\Lambda$. La topologie étant non discrète, $\pi$ est non nilpotent et l'anneau $\Lambda$ est intègre. Il s'ensuit que $\Lambda$ est un domaine d'intégrité principal à droite et à gauche, donc c'est un anneau de Ore à droite et à gauche et il admet un corps des fractions à droite et à gauche; voir [P.M.C.1 et 2]. La fonction d'ordre $o$ est la valuation associée. Par analogie avec la situation commutative, on appelle corps local, le corps des fractions d'un anneau de valuation discrète complet.

On obtient ainsi une bonne généralisation de la notion commutative d'anneau de valuation discrète complet; toutefois la définition d'une uniformisante est plus restrictive. Cependant si le corps résiduel $\Lambda / \mathfrak{R}$ est commutatif tout élément de valuation un est une uniformisante. On le vérifie en remarquant qu'alors $\forall \lambda$, $\mu \in \Lambda, \lambda \mu-\mu \lambda \in \mathfrak{T}$.

La notion suivante est de manière évidente propre au cas non commutatif.

DÉfINITION 1. On appelle déviation de commutativité associée à l'uniformisante $\pi$, l'automorphisme $s_{\pi}: \Lambda \rightarrow \Lambda$ défini par

$$
\forall \lambda \in \Lambda, \quad s_{\pi}(\lambda)=\mu \quad \text { avec } \pi \lambda=\mu \pi .
$$

Par définition de $\pi$, cet automorphisme vérifie

$$
\forall \lambda \in \Lambda, \quad o\left(s_{\pi}(\lambda)-\lambda\right) \geqslant 1
$$

Si on désigne par $s_{\pi}^{n}$ la $n$-ième puissance de composition de $s_{\pi}$, on obtient par itération

$$
\forall n>0, \forall \lambda \in \Lambda, \quad \pi^{n} \lambda=s_{\pi}^{n}(\lambda) \pi^{n} \quad \text { et } \quad o\left(s_{\pi}^{n}(\lambda)-\lambda\right) \geqslant 1
$$


Une uniformisante $\pi$ de la valuation $o$ est centrale lorsque la déviation de commutativité associée $s_{\pi}$ est l'identité. La proposition suivante caractérise l'existence d'une uniformisante centrale dans l'anneau $\Lambda$.

Proposition 1. Les propriétés suivantes sont équivalentes.

(i) L'anneau $\Lambda$ possède une uniformisante centrale.

(ii) Pour toute uniformisante $\pi$ de la valuation, $s_{\pi}$ est un automorphisme intérieur de l'anneau $\Lambda$.

(iii) Il existe une uniformisante $\pi$ de la valuation telle que $s_{\pi}$ soit un automorphisme intérieur de $\Lambda$.

La preuve est laissée au lecteur.

Donnons, en dehors du cas commutatif, quelques exemples simples d'anneaux de valuation discrète complets:

L'anneau des sèries formelles à une indéterminée $K[[X]]$ à coefficients dans un corps gauche est un anneau de valuation discrète complet avec uniformisante centrale et corps résiduel non commutatif.

De façon plus générale, soient $K$ un corps non nécessairement commutatif, $\delta$ une dérivation de $K$ et formons le "skew power series ring" $K[[X, 1, \delta]]$; voir [P.M.C.1 et 2]. C'est un anneau de valuation discrète complet de corps résiduel $K$. Le produit est obtenu à partir de la loi

$$
\forall \alpha \in K, \quad X \alpha=\alpha X+\delta(\alpha) X^{2}+\cdots+\delta^{n}(\alpha) X^{n+1}+\cdots
$$

La déviation de commutativité $s_{X}$ associée à $X$ est définie par $s_{X}=\Sigma_{n>0} \delta^{n} X^{n}$. Si $K$ est un corps commutatif et $\delta$ non-identiquement nulle, cet anneau est sans uniformisante centrale.

Pour d'autres exemples et questions connexes nous renvoyons à [R.V.1]-[R.V.4].

2. Structure des anneaux de valuation discrète complets. Anneaux de Cohen. Soient $\Lambda$ un anneau de valuation discrète complet, de corps résiduel $\Lambda / \mathfrak{N} ; K$ un système de représentants de $\Lambda / \mathfrak{T}$ dans $\Lambda$ et $\pi$ une uniformisante de la valuation. Alors, comme dans le cas commutatif, tout élément $\lambda$ de $\Lambda$ s'écrit de façon unique comme une série convergente $\lambda=\sum_{i>0} \alpha_{i} \pi^{i}$, avec $\alpha_{i} \in K$.

Deffinition 2. S'il existe dans $\Lambda$ un système de représentants $K$ de $\Lambda / \mathfrak{T}$ qui est un sous corps de $\Lambda$, on dit que $\Lambda$ est un anneau de Cohen et que $K$ est un corps de représentants, ou corps de Cohen de $\Lambda$.

Il s'ensuit que $K$ est nécessairement isomorphe à $\Lambda / \mathfrak{R}$ et en particulier $\Lambda$ et $\Lambda / \Re$ ont même caractéristique.

Soit $K$ un corps non nécessairement commutatif. Désignons par $K[[X]]$ le $K$-espace vectoriel à gauche des séries formelles en l'indéterminée $X$, considéré naturellement comme un groupe additif à opérateurs à droite dans le monoïde libre engendré par $X$. Notons $o$ la fonction d'ordre attachée à $X$ avec la convention $o(0)=+\infty$.

Soit $s: K[[X]] \rightarrow K[[X]]$ un endomorphisme du groupe additif à opérateurs à droite vérifiant les deux propriétés

$$
s(1)=1 \quad \text { et } \quad \forall \lambda \in K[[X]], \quad o(s(\lambda)-\lambda) \geqslant 1 .
$$


Alors on a pour toute série $\lambda=\sum_{i \geqslant 0} \alpha_{i} X^{i}$ où $\alpha_{i} \in K, s(\lambda)=\sum_{i>0} s\left(\alpha_{i}\right) X^{i}$. Remarquons qu'en général, même si $\alpha \in K, s(\alpha)$ est une série formelle en $X$ et non un élément de $K$.

La donnée de la fontion $s$ permet de définir un produit sur $K[[X]]$ en posant

$$
\forall \alpha \in K, \quad X \alpha=s(\alpha) X
$$

d'où $\forall \lambda \in K[[X]], X \lambda=s(\lambda) X$ et si $\lambda=\sum_{i \geqslant 0} \alpha_{i} X^{i}, \mu=\sum_{i \geqslant 0} \beta_{i} X^{i}$ alors

$$
\lambda \mu=\sum_{j>0}\left(\sum_{i=0}^{j} \alpha_{i} s^{i}\left(\beta_{j-i}\right)\right) X^{j} .
$$

Il est facile de vérifier que $s$ est une isométrie de $K[[X]]$; il s'ensuit que ce produit est continu pour la valuation définie par la fonction d'ordre; ce qui s'écrit

$$
\forall \lambda, \mu \in K[[X]], \quad o(\lambda \mu)=o(\lambda)+o(\mu) .
$$

D'autre part, ce produit est associatif si, et seulement si, la fonction $s$ est multiplicative; c'est à dire

$$
\forall \alpha, \beta \in K, \quad s(\alpha \beta)=s(\alpha) s(\beta) .
$$

Dans ces conditions, on construit ainsi sur l'espace ultramétrique des séries formelles une structure d'anneau de valuation discrète complet, en général non commutative, de corps résiduel $K$, qui est un anneau de Cohen et que l'on notera $K[[X, s]]$. Réciproquement on a le

THÉORÈME 1. Tout anneau de valuation discrète complet qui est un anneau de Cohen est isomorphe à un $K[[X, s]]$ où $K$ est un corps de Cohen, $X$ une uniformisante de la valuation et $s$ la déviation de commutativité associée $\grave{a} X$.

La vérification est laissée au lecteur.

COROllaire 1. Tout anneau de Cohen non commutatif, à corps résiduel commutatif est sans uniformisante centrale.

Un problème important est celui de savoir si, comme en algèbre commutative, tout anneau de valuation discrète complet de même caractéristique que son corps résiduel est un anneau de Cohen. Nous donnons dans le $\$ 5$ une réponse négative à cette conjecture, qui n'est pas un contre exemple isolé mais met en évidence l'existence d'une nouvelle classe d'anneaux de valuation discrète complets: ceux qui ne sont pas de Cohen en égale caractéristique.

Pour des contre exemples isolés, voir [R.V.5 et 6].

Nous terminons cette section en donnant quelques propriétés de la déviation de commutativité d'un anneau de Cohen.

Soit $K[[X, s]]$ un anneau de Cohen et posons $s=\Sigma_{n \geqslant 0} \delta_{n} X^{n}$; il est clair que la donnée de $s$ est équivalente à celle d'une suite $\left(\delta_{n}\right)_{n \geqslant 0}$ d'endomorphismes additifs de $K$, liés entre eux par des équations traduisant que $s$ est une fonction multiplicative. Notons que $\delta_{0}=1_{K}$ et que $\delta_{1}$ vérifie

$$
\forall \alpha, \beta \in K \quad \delta_{1}(\alpha \beta)=\delta_{1}(\alpha) \beta+\alpha \delta_{1}(\beta) .
$$

Pour cette raison, la suite $\left(\delta_{n}\right)_{n \geqslant 0}$ est appelée une suite de "hautes dérivations" de $K$, et $\delta_{n}$ est la dérivation d'ordre $n$. 
En particulier, si $\delta$ est une dérivation de $K$, et si on prend pour tout $n \geqslant 0: \delta_{n}=$ $\delta^{n}$ (la $n$-ième puissance de composition), alors la fonction $s=\Sigma_{n>0} \delta^{n} X^{n}$ définit une structure d'anneau de Cohen dans lequel le produit s'obtient à partir de

$$
\forall \alpha \in K, \quad X \alpha-\alpha X=X \delta(\alpha) X .
$$

En effet la fonction $s$ est égale à $1+X \delta$ et on vérifie aisément qu'elle est multiplicative.

L'anneau ainsi obtenu, noté $K[[X, \delta]]$, est appelé anneau de Cohen à dérivation. Donnons un exemple:

Soit $K$ un corps local (commutatif) d'égale caractéristique, $k$ son corps résiduel; alors pour toute uniformisante $Y$ de la valuation, $K$ s'identifie au corps des séries de Laurent: $k((Y))$. Si nous notons $D_{Y}$ la $k$-dérivation continue de $K$ qui est la dérivée par rapport à $Y$, l'anneau de Cohen à dérivation $K\left[\left[X, D_{Y}\right]\right]$ est sans uniformisante centrale. Le produit s'obtient à partir de la loi $X Y-Y X=X^{2}$; et il est facile de voir en résolvant en $\lambda$ les équations $\lambda Y=Y \lambda$ et $X \lambda=\lambda X$ qu'en caractéristique nulle le centre de cet anneau se réduit à $k$ et qu'en caractéristique $p$ première son centre est le sous anneau (commutatif) $k\left(\left(Y^{p}\right)\right)\left[\left[X^{p}\right]\right]$.

3. Anneaux de Cohen à dérivation sur un corps local. Nous nous proposons d'étudier les anneaux de Cohen à dérivation $K[[X, \delta]]$, lorsque $K$ est un corps local (commutatif), à corps résiduel $k$ de caractéristique nulle et algébriquement clos, et $\delta$ une $k$-dérivation continue de $K$ (relativement à la valuation de $K$ ); voir [J.P.S.1][J.P.S.3]. Rappelons qu'un corps local est un corps muni d'une valuation discrète, notée $\nu$, complet pour la topoplogie définie par la valuation. La caractéristique du corps résiduel étant nulle, le théorème classique de Cohen nous permet, pour chaque choix d'une uniformisante $Y$, d'identifier $K$ au corps des séries de Laurent: $k((Y))$. Si nous notons $D_{Y}$ la $k$-dérivation continue de $K$ qui est la dérivée par rapport à $Y$, l'anneau de Cohen: $k((Y))\left[\left[X, D_{Y}\right]\right]$, donné en exemple à la fin de la section précédente, servira de guide tout au long de cette étude. Le problème posé ici est de construire pour les anneaux $K[[X, \delta]]$ de "bonnes représentations" en fonction de la dérivation $\delta$. Ce travail est annoncé dans [R.V.7].

Rappelons d'abord, afin de fixer les notations, des résultats plus ou moins bien connus que l'on trouve dans [J.P.S.1]-[J.P.S.3], [A.G.], [J.P.L.].

Soit $K$ un corps local à corps résiduel $k$ de caractéristique nulle; on note par $\theta$ l'anneau des entiers de $K$ et par $\mathfrak{p}$ son idéal maximal, de sorte que $\theta / \mathfrak{p}=k$. La topologie définie par la valuation discrète sur $K$ est la topologie $\mathfrak{p}$-adique.

DÉfINITION 3. On appelle $K$-espace vectoriel des $k$-différentielles topologiques de $K$ le couple universel $\Omega_{k}(K)$, d: $K \rightarrow \Omega_{k}(K)$ constitué d'un $K$-espace vectoriel $\Omega_{k}(K)$ topologique, séparé, pour la topologie $\mathfrak{p}$-adique $\left(\mathfrak{p}^{i} d(\theta)\right)_{i>0}$ et d'une application $k$-linéaire continue $d$, vérifiant

$$
\forall \alpha, \beta \in K, \quad d \alpha \beta=d \alpha \cdot \beta+\alpha \cdot d \beta .
$$

Le lemme suivant se trouve dans [J.P.S.1].

LEMme 1. $\Omega_{k}(K)$ est un $K$-espace vectoriel de dimension un. Soit $Y$ une uniformisante de la valuation et pour tout $\alpha \in K$, notons $D_{Y} \alpha$ la dérivée par rapport à $Y$; on a alors $d \alpha=D_{Y} \alpha \cdot d Y$ et $d Y$ forme une base de $\Omega_{k}(K)$ sur $K$. 
Définition 4. On appelle $K$-espace vectoriel des $k$-dérivations continues de $K$, noté $\operatorname{Der}_{k}(K)$, l'ensemble des applications $k$-linéaires, continues, $\delta: K \rightarrow K$ vérifiant

$$
\forall \alpha, \beta \in K, \quad \delta(\alpha \beta)=\delta(\alpha) \beta+\alpha \delta(\beta) .
$$

Le lemme suivant se trouve dans [A.G., E.G.A.IV].

LEMME 2. $\operatorname{Der}_{k}(K)$ est isomorphe au dual de $\Omega_{k}(K)$

$$
\operatorname{Hom}_{K}\left(\Omega_{k}(K), K\right) \simeq \operatorname{Der}_{k}(K) .
$$

Il s'ensuit que $\operatorname{Der}_{k}(K)$ est un $K$-espace vectoriel de dimension un, et si $Y$ est une uniformisante de la valuation, $D_{Y}$ forme une base de $\operatorname{Der}_{k}(K)$ sur $K$.

DÉFINITION 5. On appelle différentielle de $K$, tout élément $\omega \in \Omega_{k}(K)$; si $Y$ est une uniformisante, il existe $\alpha \in K$ tel que $\omega=\alpha d Y$. Le coefficient de $Y^{-1}$ dans le développement de $\alpha$ en série de Laurent par rapport à $Y$ s'appelle le résidu de $\omega$ et est noté rés $\omega$. Cette notation est justifiée car le résidu de $\omega$ est indépendant du choix de $Y$. La valuation $\nu(\omega)$ de la différentielle $\omega$ est définie en accord avec la topologie $\mathfrak{p}$-adique de $\Omega_{k}(K)$ et on a $\nu(\omega)=\nu(\alpha)$, si $\omega=\alpha d Y$. Il est facile de vérifier que cette définition ne dépend pas du choix de $Y$.

Si $\delta$ désigne une $k$-dérivation continue de $K$, non identiquement nulle, on a la

Proposition 2. Il existe une correspondance bijective entre $\operatorname{Der}_{k}(K)-\{0\}$ et $\Omega_{k}(K)-\{0\}$ définie par

$\forall \delta \in \operatorname{Der}_{k}(K)-\{0\}$ posons $\omega_{\delta}=d Y / \delta(Y) \in \Omega_{k}(K)-\{0\}$.

$\forall \omega \in \Omega_{k}(K)-\{0\}, \omega=\alpha d Y$, posons $\delta_{\omega}(Y)=1 / \alpha \in \operatorname{Der}_{k}(K)-\{0\}$.

La démonstration, simple, est laissée aux soins du lecteur.

Définition 6. La différentielle $\omega_{\delta}$ définie par la Proposition 2 sera appelée la différentielle topologique associée à la $k$-dérivation continue $\delta$ de $K$.

Donnons maintenant le résultat de représentation.

THÉORÈme 2. Soient $K$ un corps local de valuation $\nu$, à corps résiduel $k$ de caractéristique nulle et algébriquement clos, et $\delta$ une $k$-dérivation continue de $K$ de différentielle associée $\omega_{\delta}$. On obtient la classification suivante:

(1) $\nu\left(\omega_{\delta}\right)>-1$ (et donc en particulier rés $\omega_{\delta}=0$ ) est équivalent à: Il existe une uniformisante $Y$ de la valuation $\nu$ telle que, si on identifie $K$ à $k((Y))$, il existe un isomorphisme d'anneaux de $K[[X, \delta]]$ sur $k\left(\left(Y^{1 /\left(\nu\left(\omega_{\delta}\right)+1\right)}\right)\right)\left[\left[X, D_{Y}\right]\right]$, qui induit l'identité sur $k$ et qui transforme $X$ en $X, Y$ en $Y^{1 /\left(\nu\left(\omega_{\delta}\right)+1\right)}$ et $\delta$ en $D_{Y}$ la dérivée par rapport à $Y$.

(2) $\nu\left(\omega_{\delta}\right)<-1$ et rés $\omega_{\delta}=0$ est équivalent à: Il existe une uniformisante $Y$ de la valuation $\nu$ telle que, si on identifie $K$ à $k((Y))$, il existe un isomorphisme d'anneaux de $K[[X, \delta]]$ sur $k\left(\left(Y^{-1 /\left(\nu\left(\omega_{\delta}\right)+1\right)}\right)\right)\left[\left[X, D_{Y^{-1}}\right]\right]$, qui induit l'identité sur $k$ et qui transforme $X$ en $X, Y$ en $Y^{-1 /\left(\nu\left(\omega_{\delta}\right)+1\right)}$ et $\delta$ en $D_{Y^{-1}}$ la dérivée par rapport à $Y^{-1}$.

(3) $\nu\left(\omega_{\delta}\right)=-1$ est équivalent $\grave{a}$ :

Il existe une uniformisante $Y$ de la valuation $\nu$ telle que, si on identifie $K$ à $k((Y))$, la $k$-dérivation $\delta$ est égale à $D_{\log Y} /$ rés $\omega_{\delta}$ où $D_{\log Y}$ désigne la dérivée par rapport au logarithme formel de $Y$. 
(4) $\nu\left(\omega_{\delta}\right)<-1$ et rés $\omega_{\delta} \neq 0$ est équivalent à:

Il existe:

* Un corps local $L$ extension continue transcendante de $K$ dont le corps résiduel l est extension transcendante pure monogène de $k$.

* Une uniformisante $Y$ de la valuation de $L$, qui n'est pas dans $K$, et qui permet d'identifier $L$ à $l((Y))$ et l à $k(\log Y)($ où $\log Y$ désigne le logarithme formel de $Y)$.

*Un prolongement continu de la $k$-dérivation $\delta$ à $L$, vérifiant $\delta(\log Y)=\delta(Y) / Y$.

Alors on peut construire un plongement d'anneaux de $K[[X, \delta]]$ dans $l\left(\left(Y^{-1 /\left(\nu\left(\omega_{6}\right)+1\right)}\right)\right)\left[\left[X, D_{Y^{-1}}\right]\right]$, qui transforme $X$ en $X$ et $\delta$ en $D_{Y^{-1 .}}$. Ce plongement est obtenu comme composé du plongement naturel de $K[[X, \delta]]$ dans $L[[X, \delta]]$ laissant $X$ fixe et de l'isomorphisme d'anneaux de $l((Y))[[X, \delta]]$ sur $l\left(\left(Y^{-1 /\left(\nu\left(\omega_{b}\right)+1\right)}\right)\right)\left[\left[X, D_{Y^{-1}}\right]\right]$ qui induit l'identité sur $l$ et qui transforme $X$ en $X, Y$ en $Y^{-1 /\left(\nu\left(\omega_{b}\right)+1\right)}$ et $\delta$ en $D_{Y^{-1}}$.

La démonstration, assez longue, se subdivise en plusieurs parties. Montrons d'abord les assertions (1) et (2) qui correspondent à une forme différentielle sans résidu.

Le corps $k$ étant de caractéristique nulle, il en est de même de $K$ et l'hypothèse rés $\omega_{\delta}=0$ implique que la différentielle topoplogique $\omega_{\delta}$ admet une primitive unique $\alpha \in K-\{0\}$ de valuation non nulle (car $\left.\nu\left(\omega_{\delta}\right) \neq-1\right)$. donc: $d \alpha=\omega_{\delta}$ et $\nu(\alpha)=\nu\left(\omega_{\delta}\right)+1$. Le corps $k$ étant algébriquement clos, il existe au moins une (et exactement $\left.\left|\nu\left(\omega_{\delta}\right)+1\right|\right)$ uniformisante $Y$ de la valuation de $K$ telle que $Y^{\nu\left(\omega_{\delta}\right)+1}=\alpha$ et $\nu(Y)=1$. On a alors

$$
\omega_{\delta}=d \alpha=d Y^{\nu\left(\omega_{\delta}\right)+1}=\left(\nu\left(\omega_{\delta}\right)+1\right) Y^{\nu\left(\omega_{\delta}\right)} d Y
$$

et donc

$$
\delta(Y)=\frac{1}{\left(\nu\left(\omega_{\delta}\right)+1\right) Y^{\nu\left(\omega_{\delta}\right)}} .
$$

Dans le cas (1), soit $k\left(\left(Y^{1 /\left(\nu\left(\omega_{\delta}\right)+1\right)}\right)\right)$ le corps des séries de Laurent en l'indéterminée $Y^{1 /\left(\nu\left(\omega_{\delta}\right)+1\right)}$ puisque $\nu\left(\omega_{\delta}\right)>-1$; il s'agit de l'extension algébrique (galoisienne) de degré $\nu\left(\omega_{\delta}\right)+1$ de $k((Y))$. La substitution de $Y$ en $Y^{1 /\left(\nu\left(\omega_{\delta}\right)+1\right)}$ définit un isomorphisme de corps valués entre $K=k((Y))$ et $k\left(\left(Y^{1 /\left(v\left(\omega_{8}\right)+1\right)}\right)\right)$, et la dérivation $\delta$ devient l'unique application fermant le diagramme:

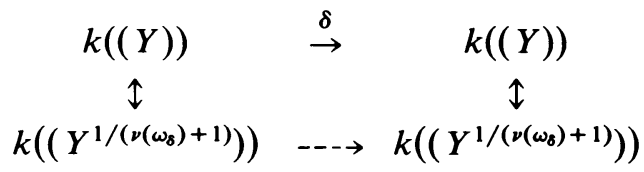

Il s'agit de la dérivation qui à $Y^{1 /\left(\nu\left(\omega_{\delta}\right)+1\right)}$ associe $1 /\left(\nu\left(\omega_{\delta}\right)+1\right) Y^{\nu\left(\omega_{\delta}\right) /\left(\nu\left(\omega_{\delta}\right)+1\right)}$ donc de la dérivation qui à $Y$ associe 1 , et on reconnait ainsi $D_{Y}$.

Dans le cas (2), puisque $\nu\left(\omega_{\delta}\right)<-1$, le corps des séries de Laurent $k\left(\left(Y^{-1 /\left(\nu\left(\omega_{\delta}\right)+1\right)}\right)\right)$ est encore une extension galoisienne de degré $-\left(\nu\left(\omega_{\delta}\right)+1\right)$ de $k((Y))$. La substitution de $Y$ en $Y^{-1 /\left(\nu\left(\omega_{b}\right)+1\right)}$ définit un isomorphisme de corps valués entre $K=k((Y))$ et $k\left(\left(Y^{-1 /\left(\nu\left(\omega_{b}\right)+1\right)}\right)\right)$, et la dérivation $\delta$ devient l'unique application qui a $Y^{1 /\left(\nu\left(\omega_{\delta}\right)+1\right)}$ associe $1 /\left(\nu\left(\omega_{\delta}\right)+1\right) Y^{-\nu\left(\omega_{\delta}\right) /\left(\nu\left(\omega_{\delta}\right)+1\right)}$ c'est à dire $D_{Y^{-1}}$. Les réciproques de (1) et de (2) s'établissent en remarquant que si $n$ est un entier 
positif la dérivation $D_{Y}$ (respectivement $\left.D_{Y^{-1}}\right)$ sur le corps local $k\left(\left(Y^{1 / n}\right)\right.$ ) admet pour différentielle associée $\omega_{D_{Y}}=d Y$ (respectivement $\omega_{D_{Y}-1}=d Y^{-1}$ ). Il s'agit d'une différentielle de valuation $n$ (respectivement de valuation $-(n+1)$ ) qui admet une primitive donc qui est sans résidu.

Montrons maintenant l'assertion (3).

Si $t$ est une uniformisante quelconque de la valuation de $K$, on peut écrire

$$
\frac{\omega_{\delta}}{\text { rés } \omega_{\delta}}=\frac{d t}{t}+\omega \text { avec } \nu(\omega) \geqslant 0 \text {. }
$$

Le corps $k$ étant de caractéristique nulle, on a comme dans la démonstration de (1) $\omega=d \alpha$ avec $\alpha \in K$ et $\nu(\alpha) \geqslant 1$. Si $e^{\alpha}$ désigne la série formelle en $t$ obtenue en substituant $\alpha$ à la variable formelle, $Y=t e^{\alpha}$ est une uniformisante de la valuation de $K$ qui vérifie $d Y=e^{\alpha} d t+t e^{\alpha} d \alpha$ et donc

$$
\frac{d Y}{Y}=\frac{\omega_{\delta}}{\text { rés } \omega_{\delta}} \text {. }
$$

Par définition de $\omega_{\delta}$, il s'ensuit que

$$
\delta(Y)=\frac{Y}{\text { rés } \omega_{\delta}} .
$$

Si on définit formellement $\log Y\left(\operatorname{par} e^{\log Y}=Y\right)$ on peut convenir de noter $D_{\log Y} Y=Y$ et $\delta$ s'écrit alors $\delta=D_{\log Y} /$ rés $\omega_{\delta}$. Réciproquement, pour tout $x \in k-\{0\}, x D_{\log Y}$ est une dérivation sur $k((Y))$ dont la différentielle associée $d Y / x Y$ est de valuation -1 .

DÉmONSTRATION DE L'ASSERTION (4). Soit $l$ une extension transcendante pure monogène de $k$ et notons par $L$ le corps des séries de Laurent à une indéterminée et à coefficients dans $l$. C'est un corps local qui peut être considéré naturellement comme une extension continue transcendante de $K$. Le corps $L$ peut aussi être obtenu en complétant une extension transcendante pure monogène de $K$ pour la valuation qui prolonge trivialement celle de $K$ (c'est à dire qui s'annule sur une base pure de l'extension; voir [N.B., Chapitre VI, §10, 1, Proposition 2]).

La valuation de $L$ sera encore notée $\nu$.

Définissons une fonction logarithme sur le corps local $L$. Si $t$ est une uniformisante de la valuation de $K$, c'est aussi une uniformisante de la valuation de $L$, et tout élément $\sigma \in L-\{0\}$ admet la représentation $\sigma=y t^{\nu(\sigma)}(1+\varepsilon)$ où $y \in l-$ $\{0\}, \varepsilon \in L$ et $\nu(\varepsilon) \geqslant 1$.

Notons $\log (1+\varepsilon)$ la série formelle classique obtenue par substitution de $\varepsilon$ à la variable formelle, c'est à dire $\sum_{i \geqslant 1}(-1)^{i-1} \varepsilon^{i} / i$ et soit $v$ une base pure de $L$ sur $K$; on a alors

LEMME 3. La fonction Log: $L-\{0\} \rightarrow L$ qui à $\sigma \in L-\{0\}$ associe $\nu(\sigma) v+$ $\log (1+\varepsilon) \in L$ est une fonction continue pour la topologie de la valuation. Cette fonction vérifie les propriétés suivantes:

$$
\begin{gathered}
\forall \sigma, \tau \in L-\{0\}, \quad \log (\sigma \tau)=\log \sigma+\log \tau, \quad \log t=v, \\
\forall y \in l-\{0\}, \quad \log y=0 .
\end{gathered}
$$

Cette fonction sera appelée fonction logarithme sur le corps local $L$. 
Preuve. Les propriétés de la fonction Log sont immédiatement vérifiées; montrons la continuité au point $\sigma \in L-\{0\}$.

$\forall \tau \in L$ tel que $\nu(\tau) \geqslant \nu(\sigma)+1$, on a

$$
\log (\sigma+\tau)-\log \sigma=\log \left(1+\frac{\tau}{\sigma}\right)=\frac{\tau}{\sigma} \sum_{i>1} \frac{(-1)^{i-1}}{i} \frac{\tau^{i-1}}{\sigma^{i-1}}
$$

et en passant aux valuations

$$
\nu(\log (\sigma+\tau)-\log \sigma)=\nu(\tau)-\nu(\sigma)
$$

ce qui achève la démonstration.

Il s'ensuit que si $t$ est une uniformisante de la valuation de $K$, le corps résiduel $l$ admet une représentation sous la forme $k(\log t)$ et le corps local $L$ une représentation sous la forme $k(\log t)((t))$.

D'après un résultat général, sur le prolongement des dérivations aux extensions transcendantes, on sait que toute $k$-dérivation continue $\delta$ de $K$ se prolonge de façon unique en une $k$-dérivation continue de $L$ en posant $\delta(\log t)=\delta(t) / t$; en particulier $D_{t}$ se prolonge à $L$ en posant $D_{t}(\log t)=1 / t$. De façon précise, si $\sigma=\sum_{i>-\infty} y_{i} t^{i} \in L$ où $y_{i} \in l$, alors

$$
\delta(\sigma)=\sum_{i>-\infty}\left(\delta\left(y_{i}\right) t^{i}+y_{i} \delta\left(t^{i}\right)\right)=\left[\sum_{i>-\infty}\left(D_{\log t}\left(y_{i}\right)+i y_{i}\right) t^{i-1}\right] \delta(t) .
$$

Il s'ensuit, par dualité, une extension du $K$-espace vectoriel des $k$-différentielles topologiques de $K$ selon le $L$-espace vectoriel des $k$-différentielles topologiques de $L: \Omega_{k}(K) \otimes_{K} L ;$ l'application différentielle, encore notée $d: L \rightarrow \Omega_{k}(K) \otimes_{K} L$, étant définie par si $\sigma=\sum_{i>-\infty} y_{i} t^{i} \in L$ où $y_{i} \in l$, alors

$$
d \sigma=\left[\sum_{i>-\infty}\left(D_{\log t}\left(y_{i}\right)+i y_{i}\right) t^{i-1}\right] d t .
$$

Si $\delta$ est une $k$-dérivation continue de $K$ et $\omega_{\delta}$ sa différentielle toppologique associée dans $\Omega_{k}(K)$, il est bien clair que dans $\Omega_{k}(K) \otimes_{K} L$, on a encore $\forall \sigma \in L$, $\omega_{\delta}=d \sigma / \delta(\sigma)$. L.

Etablissons maintenant un résultat sur certains changements d'uniformisantes de

Proposition 3. Soit $u$ une uniformisante de la valuation de $L$ du type $u=$ $x t(1+\varepsilon)$ où $x \in k-\{0\}$ et $\varepsilon \in L$ avec $\nu(\varepsilon) \geqslant 1$. Alors Log $u$ est transcendant sur $k((u))$. Le corps résiduel l est isomorphe à $k(\log u)$ et le corps local $L$ est isomorphe au corps local $k(\log u)((u))$.

Preuve. De $u=x t(1+\varepsilon)$, on déduit

$\log u=\log t+\log (1+\varepsilon)$ d'où

$$
D_{t}(\log u)=\frac{1}{t}+\frac{D_{t}(1+\varepsilon)}{1+\varepsilon}=\frac{1+\varepsilon+t D_{t}(1+\varepsilon)}{t(1+\varepsilon)} .
$$

Or $D_{t} u=x\left(1+\varepsilon+t D_{t}(1+\varepsilon)\right)$ donc

$$
D_{t}(\log u)=\frac{D_{t} u}{x t(1+\varepsilon)}=\frac{D_{t} u}{u} .
$$

Mais on sait que $D_{t}(\log u)=D_{u}(\log u) D_{t} u$ d'où $D_{u}(\log u)=1 / u$. 
$k$ étant algébriquement clos, si $\log u$ était algébrique sur $k((u))$, d'après le théorème de Puiseux, il existerait un entier $n>0$ tel que $\log u \in k\left(\left(u^{1 / n}\right)\right)$; ce qui s'écrirait

$$
\log u=\sum_{i>-\infty} x_{i} u^{i / n} \quad \text { où } x_{i} \in k
$$

d'où

$$
D_{u}(\log u)=\sum_{i>-\infty} \frac{i}{n} x_{i} u^{i / n^{-1}}=\frac{1}{u},
$$

ce qui est contradictoire.

Il s'ensuit que le plus petit sous corps de $L$ engendré par $k((u))$ et $\log u$ est l'extension transcendante pure monogène $k((u))(\log u)$. Son complété pour la topologie de la valuation de $L$ est le corps local $k(\log u)((u))$, voir [N. B., Chapitre VI]; qui est un sous corps fermé de $L$. Montrons que $k(\log u)((u))$ est dense dans $L$ ce qui achèvera de prouver qu'il coïncide avec $L$.

Puisque $u$ est une uniformisante de la valuation de $L$, on peut représenter le corps local $L$ sous la forme $k(\log t)((u))$; et il suffit de montrer que $\log t$ est limite d'une suite d'éléments de $k(\log u)((u))$ pour la topologie de la valuation de $L$. Ecrivons $t$ en fonction de $u$

$$
t=x^{-1} u(1+\eta) \quad \text { où } x^{-1} \in k-\{0\} \quad \text { et } \quad \eta \in k(\log t)((u)) \quad \text { avec } \nu(\eta) \geqslant 1 \text {. }
$$

d'où $\log t=\log u+\log (1+\eta)$.

$\log (1+\eta)$ est une série formelle en $u$ que l'on peut écrire

$$
\log (1+\eta)=\sum_{i>1} y_{i} u^{i} \quad \text { où } y_{i} \in k(\log t)
$$

et donc $\log t=\log u=\sum_{i>1} y_{i} u^{i}$.

Etablissons un lemme préliminaire.

LEMME 4. Pour tout entier $i>0$, on a l'équivalence

(i) $\exists \sigma_{i} \in k(\log u)((u))$ tel que $\nu\left(\log t-\sigma_{i}\right) \geqslant i$.

(ii) $\forall y \in k(\log t), \exists \tau_{i} \in k(\log u)((u))$ tel que $\nu\left(y-\tau_{i}\right) \geqslant i$.

Preuve. Il est bien clair qu'il suffit d'établir que (i) $\Rightarrow$ (ii). L'hypothèse $\nu\left(\log t-\sigma_{i}\right) \geqslant i$ implique $\nu\left(\sigma_{i}\right)=0$, c'est à dire $\sigma_{i} \in k(\log u)[[u]]$. Si $y$ est un élément de $k(\log t)$, convenons de noter $y\left(\sigma_{i}\right)$ sa valeur dans $k(\log u)((u))$ obtenue en substituant $\sigma_{i}$ à $\log t$.

Si $a$ est un polynome de $k[\log t]$, alors il est facile de voir que

$$
a-a\left(\sigma_{i}\right)=\left(\log t-\sigma_{i}\right) \theta \quad \text { avec } \theta \in k(\log t)[[u]]
$$

et donc $\nu\left(a-a\left(\sigma_{i}\right)\right) \geqslant \nu\left(\log t-\sigma_{i}\right) \geqslant i$.

Comme précédemment $\nu(a)=0$ implique $\nu\left(a\left(\sigma_{i}\right)\right)=0$ et en particulier $a\left(\sigma_{i}\right)$ est différent de zéro.

Si $y=a / b$ est un élément de $k(\log t)$, alors en posant $\tau_{i}=y\left(\sigma_{i}\right)$ on a

$$
y-\tau_{i}=y-y\left(\sigma_{i}\right)=\frac{a}{b}-\frac{a\left(\sigma_{i}\right)}{b\left(\sigma_{i}\right)}=\frac{\left(a-a\left(\sigma_{i}\right)\right) b\left(\sigma_{i}\right)-a\left(\sigma_{i}\right)\left(b-b\left(\sigma_{i}\right)\right)}{b b\left(\sigma_{i}\right)} .
$$


Passons aux valuations et utilisons l'inégalité ultramétrique

$$
\nu\left(y-\tau_{i}\right) \geqslant \operatorname{Min}\left\{\nu\left(\frac{1}{b}\left(a-a\left(\sigma_{i}\right)\right)\right), \nu\left(\frac{a\left(\sigma_{i}\right)}{b b\left(\sigma_{i}\right)}\left(b-b\left(\sigma_{i}\right)\right)\right)\right\}
$$

or

$$
\nu\left(\frac{1}{b}\right)=\nu\left(\frac{a\left(\sigma_{i}\right)}{b b\left(\sigma_{i}\right)}\right)=0
$$

d'où $\nu\left(y-\tau_{i}\right) \geqslant i$, ce qui achève la preuve du lemme. Afin de terminer la démonstration de la proposition, nous montrons, par récurrence sur l'entier $j$, que l'on peut approximer $\log t$ par une suite $\left(\sigma_{j}\right)_{j \geqslant 1}$ vérifiant $\nu\left(\log t-\sigma_{j}\right) \geqslant j$ où $\sigma_{j} \in k(\log u)((u))$.

Si $j=1$, la formule $\log t=\log u+\sum_{i>1} y_{i} u^{i}$ montre que l'on peut prendre $\sigma_{1}=\log u$.

Supposons que pour tout $i<j$, il existe $\sigma_{i} \in k(\log u)((u))$ vérifiant $\nu\left(\log t-\sigma_{i}\right)$ $\geqslant i$. Alors de $v\left(\log t-\left(\log u+\sum_{i=1}^{j-1} y_{i} u^{i}\right)\right) \geqslant j$ et en utilisant le lemme précédent sous la forme $\forall i<j, \exists \tau_{i} \in k(\log u)((u))$ tel que $\nu\left(y_{j-i}-\tau_{i}\right) \geqslant i$, on peut construire $\sigma_{j} \in k(\log u)((u))$ en posant

$$
\sigma_{j}=\log u+\sum_{i=1}^{j-1} \tau_{j-i} u^{i}
$$

et on a bien $\nu\left(\log t-\sigma_{j}\right) \geqslant j$.

Contruisons une bonne uniformisante $Y$ de la valuation de $L$, qui n'appartient pas à $K$ et à laquelle nous appliquerons les résultats obtenus sur les changements d'uniformisantes.

L'hypothèse de l'assertion (4), $\nu\left(\omega_{\delta}\right)<-1$ et rés $\omega_{\delta} \neq 0$, nous permet d'écrire la différentielle topologique $\omega_{\delta}$ sous la forme

$$
\omega_{\delta}=\left(\text { rés } \omega_{\delta}\right) \frac{d t}{t}+\omega
$$

où $t$ est une uniformisante de la valuation de $K$, et $\omega$ une différentielle topologique vérifiant $\nu(\omega)=\nu\left(\omega_{\delta}\right)<-1$ et rés $\omega=0$. Le corps $k$ étant de caractéristique nulle, il existe $\alpha \in K-\{0\}$ tel que $d \alpha=\omega$ et $\nu(\alpha)=\nu\left(\omega_{\delta}\right)+1<0$. La différentielle $\omega_{\delta}$ qui ne s'intègre pas dans $K$, admet une primitive $\sigma$ dans $L$, représenté sous la forme $k(\log t)((t))$, en posant: $\sigma=\left(\right.$ rés $\left.\omega_{\delta}\right) \log t+\alpha$. On a $d \sigma=\omega_{\delta}$ et $\nu(\sigma)=\nu(\alpha)=$ $\nu\left(\omega_{\delta}\right)+1<0$.

Le corps $k$ étant algébriquement clos, il est facile de voir qu'il existe une uniformisante $Y$ de la valuation de $L$, telle que $Y^{\nu\left(\omega_{\delta}\right)+1}=\sigma . Y$ est du type $Y=x t(1+\varepsilon)$ où $x \in k-\{0\}$ et $\varepsilon \in L$ avec $\nu(\varepsilon) \geqslant 1$. $Y$ n'appartient pas à $K$, car $\sigma$ n'appartient pas à $K$ (on peut aussi voir directement que $\varepsilon$ n'appartient pas à $K$ ).

De façon analogue aux démonstrations des précédentes assertions, on a

$$
\omega_{\delta}=d \sigma=d Y^{\nu\left(\omega_{\delta}\right)+1}=\left(\nu\left(\omega_{\delta}\right)+1\right) Y^{\nu\left(\omega_{\delta}\right)} d Y
$$

avec $d Y \in \Omega_{k}(K) \otimes_{K} L$ et en utilisant notre remarque sur le prolongement des dérivations et des différentielles topologiques

$$
\delta(Y)=\frac{1}{\left(\nu\left(\omega_{\delta}\right)+1\right) Y^{\nu\left(\omega_{\delta}\right)}} .
$$


La Proposition 3 que nous venons d'établir nous permet d'identifier $L$ à $k(\log Y)((Y))$ notée $l((Y))$. Le corps des séries de Laurent $l\left(\left(Y^{-1 /\left(\nu\left(\omega_{\delta}\right)+1\right)}\right)\right)$ est une extension galoisienne de degré $-\left(\nu\left(\omega_{\delta}\right)+1\right)$ de $l((Y))$ et la substitution de $Y$ en $Y^{-1 /\left(\nu\left(\omega_{\delta}\right)+1\right)}$ définit un isomorphisme de corps valués entre $l((Y))$ et $l\left(\left(Y^{-1 /\left(\nu\left(\omega_{\delta}\right)+1\right)}\right)\right)$. La dérivation $\delta$ devient par cet isomorphisme l'application $D_{Y^{-1}}$.

Remarquons que la substitution de $Y$ en $Y^{-1 /\left(\nu\left(\omega_{b}\right)+1\right)}$ ne change pas $l$ car $k(\log Y)=k\left(\log \left(Y^{-1 /\left(\nu\left(\omega_{0}\right)+1\right)}\right)\right)$.

La réciproque est immédiate; si $n$ est un entier positif, la dérivation $D_{Y^{-1}}$ sur le corps local $k(\log Y)\left(\left(Y^{1 / n}\right)\right)$, qui est supposée être le prolongement d'une $k$ dérivation continue de $K$ avec $Y \notin K$, admet pour différentielle associée $\omega_{D_{Y^{-1}}}=$ $d Y^{-1}$ (on suppose donc que $\omega_{D_{Y^{-1}}} \in \Omega_{k}(K)$ ). Il s'agit d'une différentielle de valuation $-(n+1)$ dont la primitive $Y^{-1}$ n'appartient pas à $K$; il s'ensuit que cette différentielle possède un résidu différent de zéro.

4. Application à un cas de valuation dense: corps de Puiseux. Si $K$ est un corps local, à corps résiduel $k$ de caractéristique nulle et algébriquement clos, sa clôture algébrique $\bar{K}$ définie à un isomorphisme près est appelée corps de Puiseux du corps $K$. On sait, voir [J.P.S.3], que pour chaque choix d'une uniformisante $Y$ de la valuation de $K$, on a la représentation $\bar{K}=\cup_{i>0} k\left(\left(Y^{1 / i}\right)\right)$. On introduit ainsi, pour chaque entier $i$ positif le corps local $K_{i}=k\left(\left(Y^{1 / i}\right)\right)$, défini à un isomorphisme près (et qui donc ne dépend pas de $Y$ ), extension galoisienne de degré $i$ de $K$; et on a $\bar{K}=\cup_{i>0} K_{i}$. On considère $\bar{K}$ comme un corps de valuation dense, muni de la valuation $\nu$, qui prolonge celle des différents $K_{i}$ et on se propose d'étendre à l'anneau $\bar{K}[[X, \delta]]$, où $\delta$ est une $k$-dérivation continue de $\bar{K}$, le résultat obtenu dans le paragraphe précédent.

Donnons d'abord quelques précisions sur les $k$-dérivations continues et sur les $k$-différentielles topologiques de $\bar{K}$. On sait par un résultat général sur le prolongement des dérivations dans les extensions algébriques séparables, que tout élément d'un $\operatorname{Der}_{k}\left(K_{i}\right)$ se prolonge de façon unique en un élément de $\operatorname{Der}_{k}(\bar{K})$. On se propose d'établir ici la réciproque, à savoir que l'on atteint par ce procédé tous les élément de $\operatorname{Der}_{k}(\bar{K})$.

Lemme 5. Toute dérivation continue $\delta$ de $\bar{K}$ est le prolongement unique à $\bar{K} d$ 'une dérivation continue d'un $K_{n}$ pour $n$ entier convenable.

Preuve. Si $\delta \in \operatorname{Der}_{k}(\bar{K})$, il existe un entier $n$ tel que $\delta(Y) \in K_{n}$; comme $\delta$ est une dérivation

$$
\delta\left(Y^{1 / n}\right)=\frac{1}{n} Y^{1 / n-1} \delta(Y)=\frac{1}{n} Y^{(1-n) / n} \delta(Y)
$$

et donc $\delta\left(Y^{1 / n}\right) \in K_{n}$; il s'ensuit que la restriction de $\delta$ à $K_{n}$ appartient à $\operatorname{Der}_{k}\left(K_{n}\right)$ et $\delta$ en est l'unique prolongement dans $\operatorname{Der}_{k}(\bar{K})$.

A chaque corps local $K_{i}$, on associe le $K_{i}$-espace vectoriel de dimension un des $k$-différentielles topologiques de $K_{i}$; il s'agit du couple $\Omega_{k}\left(K_{i}\right), d_{i}: K_{i} \rightarrow \Omega_{k}\left(K_{i}\right)$.

Les $\Omega_{k}\left(K_{i}\right)$ forment un système inductif filtrant dont les flèches de transition sont des injections. Il est naturel, voir [J.P.L.], de définir $\Omega_{k}(\bar{K})$ comme la limite 
inductive des tensorisés

$$
\Omega_{k}(\bar{K})=\underset{i>0}{\operatorname{Lim}}\left(\Omega_{k}\left(K_{i}\right) \otimes_{K_{i}} \bar{K}\right)
$$

et il est facile de remarquer (en utilisant le Lemme 5 et un argument de dualité) que pour chaque entier $i>0$

$$
\Omega_{k}\left(K_{i}\right) \otimes_{K_{i}} \bar{K} \simeq \Omega_{k}(\bar{K}) .
$$

Il s'ensuit que $\Omega_{k}(\bar{K})$ est un $\bar{K}$-espace vectoriel de dimension un et on notera $d: \bar{K} \rightarrow \Omega_{k}(\bar{K})$ l'application $k$-linéaire. De plus les injections de $K_{i}$-espaces vectoriels

$$
\Omega_{k}\left(K_{i}\right) \hookrightarrow \Omega_{k}\left(K_{i}\right) \otimes_{K_{i}} \bar{K}
$$

passent à la limite inductive selon un isomorphisme de $\bar{K}$-espace vectoriel, et donc $\Omega_{k}(\bar{K}) \simeq \cup_{i>0} \Omega_{k}\left(K_{i}\right)$. Définissons maintenant la valuation et le résidu d'une différentielle de $\bar{K}$.

On munit $\Omega_{k}(\bar{K})$ de la valuation dense $\nu$ définie de la manière suivante: si $Y$ est une uniformisante de la valuation de $K$ et $\omega \in \Omega_{k}(\bar{K})$, puisque $d Y$ est une base de $\Omega_{k}(\bar{K})$ comme $\bar{K}$-espace vectoriel, on a $\omega=\alpha d Y$ où $\alpha$ est unique dans $\bar{K}$ et on pose $\nu(\omega)=\nu(\alpha)$. (On vérifie que cela ne dépend pas de $Y$.)

Remarquons qu'il existe au moins un entier $n>0$ tel que $\alpha \in K_{n}$ et donc $\omega \in \Omega_{k}\left(K_{n}\right)$. Considérant $K_{n}$ comme un corps local, notons $\nu_{n}(\alpha)$ (respectivement $\left.\nu_{n}(\omega)\right)$ la valuation entière de $\alpha$, (respectivement de $\omega$ ) dans $K_{n}$ (respectivement dans $\Omega_{k}\left(K_{n}\right)$ ); on a $\nu_{n}(\alpha)=n \nu(\alpha)=n \nu(\omega)$ et de $\omega=\alpha d Y=n Y^{(n-1) / n} \alpha d Y^{1 / n}$ on déduit $\nu_{n}(\omega)=n-1+\nu_{n}(\alpha)$ d'où $\nu_{n}(\omega) / n+1 / n=\nu(\omega)+1$. Ceci permet de définir le résidu de $\omega \in \Omega_{k}(\bar{K})$, noté rés $\omega$, comme le coefficient de $Y^{-1}$ dans le développement de $\alpha$ selon les puissances rationnelles de $Y$, car si on note rés ${ }_{n} \omega$ le résidu de $\omega$ dans $\Omega_{k}\left(K_{n}\right)$ on a

$$
\begin{aligned}
\text { rés }_{n} \omega & =\text { coefficient de } Y^{-1 / n} \text { du développement de } n Y^{(n-1) / n} \alpha \text { dans } K_{n} \\
& =n\left(\text { coefficient de } Y^{-1} \text { du développement de } \alpha \text { dans } \bar{K}\right)
\end{aligned}
$$

d'où rés $\omega=$ rés $_{n} \omega / n$.

Si maintenant $\delta$ est une $k$-dérivation continue, non nulle, de $\bar{K}$, on lui associe de façon bijective la différentielle non nulle $\omega_{\delta}$ par $\omega_{\delta}=d Y / \delta(Y)$ où $Y$ est une uniformisante de la valuation de $K$; et on a le résultat qui généralise les assertions (1) et (2) du Théorème 2:

ThÉORème 3. Soient $\bar{K}$ le corps de Puiseux $d$ 'un corps local $K$, à corps résiduel $k$ de caractéristique nulle et algébriquement clos, et $\delta$ une $k$-dérivation continue de $\bar{K}$ de différentielle associée $\omega_{\delta}$.

Les assertions (1) et (2) sont équivalentes:

(1) $\nu\left(\omega_{\delta}\right)>-1$ (et donc en particulier rés $\left.\omega_{\delta}=0\right)$.

(2) Il existe une uniformisante $Y$ de la valuation de $K$ telle que, si on identifie $\bar{K} \dot{a}$ $\cup_{i>0} k\left(\left(Y^{1 / i}\right)\right)$, il existe un isomorphisme d'anneaux de $\bar{K}[[X, \delta]]$ sur $\bar{K}\left[\left[X, D_{Y}\right]\right]$ qui induit l'identité sur $k$ et qui transforme $X$ en $X, Y$ en $Y^{1 /\left(v\left(\omega_{\delta}\right)+1\right)}$ et $\delta$ en $D_{Y}$ la dérivée par rapport à $Y$. 
Les assertions (3) and (4) sont équivalentes:

(3) $\nu\left(\omega_{\delta}\right)<-1$ et rés $\omega_{\delta}=0$.

(4) Il existe une uniformisante $Y$ de la valuation de $K$ telle que, si on identifie $\bar{K} \dot{a}$ $\cup_{i>0} k\left(\left(Y^{1 / i}\right)\right)$, il existe un isomorphisme d'anneaux de $\bar{K}[[X, \delta]]$ sur $\bar{K}\left[\left[X, D_{Y^{-1}}\right]\right]$ qui induit l'identité sur $k$ et qui transforme $X$ en $X, Y$ en $Y^{-1 /\left(\nu\left(\omega_{\delta}\right)+1\right)}$ et $\delta$ en $D_{Y^{-1}}$ la dérivée par rapport à $Y^{-1}$.

La preuve utilise le Théorème 2 précédent; elle est laissée au lecteur.

5. Anneaux sans corps de Cohen en égale caractéristique. Nous nous proposons de donner ici l'idée d'une méthode assez générale de construction, en égale caractéristique (nulle et première), d'anneaux sans corps de Cohen.

Soient $K$ un corps, non nécessairement commutatif, et $\delta$ une dérivation de $K$. Associons le corps local, en général non commutatif, $K((X, \delta))$, corps des fractions de l'anneau de Cohen à dérivation $K[[X, \delta]]$. Ce corps est constitué des séries de Laurent en la variable $X$, et le produit est obtenu à partir de la loi $\forall \alpha \in K$, $X \alpha-\alpha X=X \delta(\alpha) X$.

Remarquons que la dérivation $\delta$ de $K$ se prolonge à $K((X, \delta))$ selon une dérivation intérieure (induite $\operatorname{par} X^{-1}$ ).

Ce procédé permet, en algèbre non commutative, d'associer à un corps muni d'une dérivation, une extension transcendante de ce corps selon un corps local, en général non commutatif. Répétons ce procédé. A partir d'un corps $K_{0}$ muni d'une dérivation $d_{0}$, on construit une suite d'extensions transcendantes de corps locaux

$$
K_{0} \subset K_{1} \subset K_{2} \subset \cdots \subset K_{n} \subset K_{n+1} \subset \cdots .
$$

à condition de se donner chaque fois une dérivation $d_{n}$ sur le corps $K_{n}$. On a pour tout entier $n \geqslant 0, K_{n+1}=K_{n}\left(\left(X_{n}, d_{n}\right)\right)$. Le corps $K_{n+1}$ peut aussi être considéré comme un corps (différentiel) de séries de Laurent en variables non commutatives, à coefficients dans $K_{0}$, dans lequel le produit se calcule grace aux dérivations $d_{0}$, $d_{1}, \ldots, d_{n}$; ce que l'on notera $K_{n+1}=K_{0}\left(\left(X_{0}, X_{1}, \ldots, X_{n} ; d_{0}, d_{1}, \ldots, d_{n}\right)\right)$.

Les anneaux de valuation discrète complets contenus dans $K_{n+1}$ et admettant $K_{n+1}$ pour corps des fractions correspondent bijectivement à certaines valuations "bien faites" de ce corps. Moyennant certaines hypothèses sur $K_{0}$, et sur $d_{0}, d_{1}, \ldots, d_{n}$, on peut ainsi construire, en égale caractéristique, des anneaux sans corps de Cohen. Donnons deux exemples.

(i) Anneau sans corps de Cohen, avec uniformisante centrale et corps résiduel commutatif. Prendre $K_{0}=k$ corps commutatif; $d_{0}=d_{1}=0$ et $d_{2}$ dérivation de $K_{2}=k\left(\left(X_{0}, X_{1}\right)\right)$ vérifiant $d_{2}\left(X_{0}\right)=X_{1}, d_{2}\left(X_{1}\right)=0$. Dans le corps $K_{3}=$ $k\left(\left(X_{0}, X_{1}, X_{2} ; 0,0, d_{2}\right)\right)$ considérons la valuation $v_{1}$ qui est la fonction d'ordre attachée à $X_{1}$ et notons $\Lambda_{1}$ l'anneau de cette valuation. Cet anneau est non commutatif car $X_{2} X_{0}-X_{0} X_{2}=X_{1} X_{2}^{2}$, avec uniformisante centrale $X_{1}$, et son corps résiduel est isomorphe au corps commutatif $k\left(\left(x_{0}, x_{2}\right)\right.$ ) (où $x_{0}$ et $x_{2}$ sont les classes de $X_{0}$ et $X_{2}$ modulo $\left.X_{1}\right)$. $\Lambda_{1}$ n'admet pas de corps de Cohen.

(ii) Anneau sans corps de Cohen, sans uniformisante centrale et corps résiduel commutatif. Nous nous contentons d'indiquer la méthode; les vérifications, même non évidentes, sont laissées au lecteur. Prendre $K_{0}=k$ corps commutatif; $d_{0}=0$, 
$d_{1}=D_{X_{0}}$ (dérivée par rapport à $\left.X_{0}\right)$ et $d_{2}$ dérivation de $K_{2}=k\left(\left(X_{0}\right)\right)\left(\left(X_{1}, D_{X_{0}}\right)\right)$ vérifiant $d_{2}\left(X_{0}\right)=X_{1}, d_{2}\left(X_{1}\right)=0$.

Dans le corps $K_{3}=k\left(\left(X_{0}, X_{1}, X_{2} ; 0, D_{X_{0}}, d_{2}\right)\right)$ considérons la valuation $v_{1}$ qui est la fonction d'ordre attachée à $X_{1}$ et $\Lambda_{1}$ l'anneau de cette valuation. Cet anneau est non commutatif $X_{1} X_{0}-X_{0} X_{1}=X_{1}^{2}$ et $X_{2} X_{0}-X_{0} X_{2}=X_{1} X_{2}^{2}$, sans uniformisante centrale, et son corps résiduel est isomorphe, comme précédemment, au corps commutatif $k\left(\left(x_{0}, x_{2}\right)\right)$. On peut montrer que $\Lambda_{1}$ ne possède pas de corps de Cohen.

Appendice. Problèmes ouverts. 1. On peut généraliser l'étude faite ici en remplaçant dans la définition d'un anneau de valuation discrète complet le centralisateur de $\Re / \Re^{2}$ noté $Z_{\Lambda}\left(\mathscr{N} / \Re^{2}\right)$ par

$$
Z_{\Lambda, \tau}\left(\mathfrak{\Re} / \mathscr{N}^{2}\right)=\left\{x \in\left(\mathscr{\Re} / \mathscr{N}^{2}\right) / \tau(\lambda) x=x \lambda, \forall \lambda \in \Lambda\right\}
$$

où $\tau$ est un automorphisme de $\Lambda$.

Si $K$ est un corps, $\tau$ un automorphisme de $K, \delta$ une dérivation de $K$, le "skew power series ring" $K[[X, \tau, \delta]]$ dans lequel le produit est défini par $\forall \alpha \in K$, $X \alpha=\tau(\alpha) X+X \delta(\alpha) X$ soit encore

$$
\begin{aligned}
& \forall \alpha \in K, \quad X \alpha=\tau(\alpha) X+\tau(\delta(\alpha)) X^{2} \\
& +\tau\left(\delta^{2}(\alpha)\right) X^{3}+\cdots+\tau\left(\delta^{i-1}(\alpha)\right) X^{i}+\cdots
\end{aligned}
$$

est un exemple qui illustre la situation nouvelle. Voir [P.M.C. 1,2].

2. En ce qui concerne les anneaux de valuation discrète complets qui ne sont pas des anneaux de Cohen, le problème reste entièrement ouvert en inégale caractéristique. L'analogue commutatif est constitué par l'étude des anneaux de vecteurs de Witt.

3. Dans le $\S 3, K$ désigne un corps local à corps résiduel $k$ de caractéristique nulle et algébriquement clos et $\delta$ une $k$-dérivation continue de $K$. La classification du Théorème 2 donne l'échantillonnage des anneaux de Cohen à dérivation que l'on peut construire sur l'espace ultramétrique complet des séries formelles $K[[X]]$. Ce problème complètement élucidé en caractéristique nulle, reste posé en caractéristique première. La difficulté provient de la perte du moyen "transcendant" qu'est le calcul de la primitive d'une forme différentielle.

Toutefois les différentes structures développées dans le Théorème 2 peuvent se déduire les unes des autres par des isomorphismes d'anneaux; comme le montre la proposition suivante:

Proposition 4. Soient $K$ un corps local à corps résiduel $k$ de caractéristique nulle et algébriquement clos et $\delta$ une $k$-dérivation continue de $K$. Pour chaque uniformisante $t$ de la valuation de $K$, l'anneau de Cohen à dérivation $K[[X, \delta]]$ est isomorphe à l'anneau $K\left[\left[Z, D_{t}\right]\right]$ où $Z$ est une indéterminée et $D_{t}$ désigne la dérivée par rapport à $t$.

Preuve. Identifions $K$ au corps $k((t))$, des séries de Laurent; le Lemme 2 du $\$ 3$ nous permet d'écrire

$$
\forall \alpha \in K \quad \delta(\alpha)=\delta(t) D_{t} \alpha
$$


On construit un homomorphisme d'anneau de $K[[X, \delta]]$ vers $K\left[\left[Z, D_{t}\right]\right]$ en substituant dans toute série formelle du premier anneau $Z / \delta(t)$ à la variable formelle $X$. On obtient ainsi une série formelle en $Z$ que l'on calcule avec la règle du produit du deuxième anneau

$$
\forall \alpha \in K \quad X \alpha-\alpha X=X \delta(\alpha) X=X \delta(t) D_{t} \alpha X
$$

substituons, il vient

$$
\frac{1}{\delta(t)} Z \alpha-\frac{1}{\delta(t)} \alpha Z=\frac{1}{\delta(t)} Z D_{t} \alpha Z
$$

d'où $Z \alpha-\alpha Z=Z D_{t} \alpha Z$.

Il est clair que cet homomorphisme est un isomorphisme d'anneau; son inverse est obtenu à partir de la substitution $Z=\delta(t) X$.

4. Aucun travail n'a été encore entrepris sur les anneaux de Cohen à "hautes dérivations" du §2. Pour l'anneau $K[[X, s]]$ il s'agit du cas où l'automorphisme $s$ est le plus général possible $s=\Sigma_{n>0} \delta_{n} X^{n}$ et les $\delta_{n}$ ne sont plus engendrées par une dérivation $\delta$, mais simplement liées par des équations traduisant que $s$ est multiplicatif. Lorsque $K$ est un corps local à corps résiduel $k$ de caractéristique nulle et algébriquement clos et les $\delta_{n}$ des hautes dérivations $k$-linéaires et continues pour la topologie de $K$, certains anneaux $K[[X, s]]$ sont isomorphes à un anneau à dérivation $K\left[\left[Z, D_{Y}\right]\right]$, et d'autres pas. L'isomorphisme, lorsqu'il existe, est obtenu par construction d'une bonne uniformisante $Z$ de la valuation de façon analogue à celle de la Proposition 4. Il serait intéressant d'entreprendre une étude fine dans cette direction. Donnons deux exemples.

(i) L'anneau $k((Y))[[X]]$ muni du produit défini par

$$
X Y-Y X=X^{2}+X^{4}+\cdots+X^{2 i}+\cdots
$$

est isomorphe à un anneau à dérivation. En effet $X Y-Y X-X(X Y-Y X) X=$ $X^{2}$ ce qui s'écrit $X Y\left(1+X^{2}\right)-\left(1+X^{2}\right) Y X=X^{2}$ et en multipliant des deux côtés par la série entière $1 /\left(1+X^{2}\right)$, on obtient

$$
\frac{X}{1+X^{2}} Y-Y \frac{X}{1+X^{2}}=\left[\frac{X}{1+X^{2}}\right]^{2} .
$$

La bonne uniformisante est $Z=X /\left(1+X^{2}\right)$.

(ii) L'anneau $k((Y))[[X]]$ muni du produit défini par $X Y-Y X=X^{2}+X^{3}$ n'est pas isomorphe à un anneau à dérivation.

La démonstration procède par l'absurde. S'il existe une uniformisante $Z$ telle que $Z Y-Y Z=Z^{2}$ alors on peut écrire $X=u Z$ où $u$ est une série formelle inversible; d'où

$$
u Z Y-Y u Z=(1+u Z) u Z u Z .
$$

Dans l'anneau $k((Y))\left[\left[Z, D_{Y}\right]\right]$ il est facile d'établir

$$
u Y-Y u=\frac{d u}{d Z} Z^{2}, \quad Z u-u Z=Z \frac{d u}{d Y} Z
$$


où $d u / d Z$ (respectivement $d u / d Y$ ) désigne la dérivée première formelle de $u$ par rapport à $Z$ (respectivement $Y$ ) d'où

$$
u(Z Y-Y Z)+\frac{d u}{d Z} Z^{3}=(1+u Z)\left(u^{2}+u Z \frac{d u}{d Y}\right) Z^{2}
$$

ce qui s'écrit, après simplification $\operatorname{par} Z^{2}$

$$
u+\frac{d u}{d Z} Z=(1+u Z)\left(u^{2}+u Z \frac{d u}{d Y}\right)
$$

Posons $u=\Sigma_{n>0} \alpha_{n} Z^{n}$ où $\alpha_{n} \in k((Y))$. L'équation précédente donne pour les termes de valuation nulle en $Z \alpha_{0}=\alpha_{0}^{2}$ d'où $\alpha_{0}=1\left(\right.$ car $\left.\alpha_{0} \neq 0\right)$ et pour les termes de valuation un en $Z 2 \alpha_{1}=2 \alpha_{1}+1$, ce qui est impossible!

\section{BIBLIOGRAPHIE}

[M.A.] M. Artin, On azumaya algebras and finite dimensional representations of rings, J. Algebra 11 (1969).

[N.B.] N. Bourbaki, Algèbre commutative, Hermann, Paris, 1964.

[P.M.C.1] P. M. Cohn, Free rings and their relations, Academic Press, New York, 1971.

[P.M.C.2] _ Skewfield constructions, Cambridge Univ. Press, London, 1977.

[A.G.] A. Grothendieck, Eléments de géométrie algébrique. IV, Inst. Hautes Etudes Sci. Publ. Math. 20 (1964).

[J.P.L.] J. P. Lafon, Algèbre commutative, Hermann, Paris, 1977.

[P.S.-O.Z.] P. Samuel et O. Zariski, Commutative algebra. Vols. I, II, Van Nostrand, New York, 1958.

[J.P.S.1] J. P. Serre, Groupes algébriques et corps de classes, Hermann, Paris, 1959.

[J.P.S.2] _ Sur les corps locaux à corps résiduel algébriquement clos, Bull. Soc. Math. France 89 (1961).

[J.P.S.3] _ Corps locaux, Hermann, Paris, 1962.

[R.V.1] R. Vidal, Anneau principal non nécessairement commutatif, C. R. Acad. Sci. Paris Sér. A-B 282 (1976).

[R.V.2] , Anneaux principaux, non nécessairement commutatifs, séparés et complets pour la topologie de Krull, C. R. Acad. Sci Sér. A-B 283 (1976).

[R.V.3] _ Anneaux de valuation discrète, complets, non nécessairement commutatifs, C. R. Acad. Sci. Paris Sér. A-B 283 (1976).

[R.V.4] _ Représentation d'anneaux principaux, non nécessairement commutatifs, séparés et complets pour la topologie de Krull, Colloq. d'algèbre commutative de l'Univ. Rennes (1976), U. E. R. Mathématiques et Informatique, Rennes, 1976.

[R.V.5] Contre exemple, non commutatif, dans la théorie des anneaux de Cohen, C. R. Acad. Sci. Paris Sér. A-B 284 (1977).

[R.V.6 _ Un exemple d'anneau de valuation discrète complet, non commutatif qui n'est pas un anneau de Cohen, C. R. Acad. Sci. Paris. Sér. A-B 284 (1977).

[R.V.7] , Anneaux de Cohen, non commutatifs, sur un corps local, C. R. Acad. Sci. Paris Sér. A-B 288 (1979).

Université de Clermont II, U.E.R. Sciences, Mathématiques Pures, 63170-Aubiere, France Current address: Flat, 63500 Issoire, France 